\title{
Presence of Skin $\alpha$-Synuclein Deposits Discriminates Parkinson's Disease from Progressive Supranuclear Palsy and Corticobasal Syndrome
}

\author{
Maria Pia Giannoccaro ${ }^{\mathrm{a}, \mathrm{b}, \mathrm{c}, *, 1}$, Patrizia Avoni ${ }^{\mathrm{a}, \mathrm{c}, 1}$, Giovanni Rizzo $^{\mathrm{c}}$, Alex Incensi ${ }^{\mathrm{b}}$, Rossella Infante ${ }^{\mathrm{a}}$, \\ Vincenzo Donadio $^{\mathrm{b}, \mathrm{c}, 1}$ and Rocco Liguori ${ }^{\mathrm{a}, \mathrm{b}, \mathrm{c}, 1}$ \\ ${ }^{\mathrm{a}}$ Department of Biomedical and Neuromotor Sciences, University of Bologna, Italy \\ ${ }^{\mathrm{b}}$ IRCCS Istituto delle Scienze Neurologiche di Bologna, Programma di Patologia Neuromuscolare e \\ Neuroimmunologia, Bologna, Italy \\ ${ }^{\mathrm{c}}$ IRCCS Istituto delle Scienze Neurologiche di Bologna, UOC Clinica Neurologica, Bologna, Italy
}

Accepted 10 November 2021

Pre-press 1 December 2021

\begin{abstract}
.
Background: Previous studies reported skin phosphorylated $\alpha$-synuclein (p-syn) deposits in Parkinson's disease (PD) patients but not in patients with parkinsonism due to tauopathies, although data on the latter are limited.

Objective: We aimed to assess the presence of skin p-syn deposits in patients with clinical diagnosis of parkinsonism usually due to tauopathy and PD.

Methods: We consecutively recruited 26 patients, 18 fulfilling clinical diagnostic criteria of progressive supranuclear palsy (PSP) and 8 of corticobasal syndrome (CBS), 26 patients with PD, and 26 healthy controls (HC). All subjects underwent skin biopsy to study p-syn deposits in skin nerves by immunofluorescence.

Results: Skin p-syn deposits were present in only two of the PSP/CBS patients and none of the HC. Conversely, all PD patients showed $\mathrm{p}$-syn deposition $(p<0.001$, Chi-square). The two $\mathrm{p}$-syn positive patients were diagnosed with PSP and CBS, respectively. Although clinical and MRI findings supported these diagnoses, both patients had some atypical features more typical of synucleinopathies.

Conclusion: The detection of skin p-syn deposits may help in the differential diagnosis of parkinsonism. Indeed, in this study, all PD patients and only two out of 26 with a clinical diagnosis of PSP/CBS had skin p-syn deposits. Furthermore, these two patients showed clinical features that could suggest an atypical synucleinopathy presentation or a mixed pathology.
\end{abstract}

Keywords: Alpha-synuclein, biomarker, corticobasal syndrome, Parkinson's disease, parkinsonism, progressive supranuclear paly, skin biopsy

\footnotetext{
${ }^{1}$ These authors contributed equally to this work.

*Correspondence to: Maria Pia Giannoccaro, IRCCS Istituto delle Scienze Neurologiche di Bologna, Via Altura 3, 40137, Bologna, Italy. Tel.: +390514966112; E-mail: mpgiannoccaro@ gmail.com.
}

\section{INTRODUCTION}

The accumulation and aggregation of one or more misfolded proteins are the common features of many neurodegenerative disorders, each one characterized by a predominant aggregated protein which represents the pathological hallmark of the disease. Idiopathic Parkinson's disease (PD) is characterized by the presence of neuronal deposits of phosphorylated 
$\alpha$-synuclein (p-syn) that in the brain aggregate into Lewy bodies [1]. Currently, the diagnosis of PD is based on consensus clinical criteria [2]. However, the cardinal parkinsonian symptoms are shared with other synucleinopathies, as well as with atypical parkinsonism, including progressive supranuclear palsy (PSP) and corticobasal syndrome (CBS). Despite the clinical overlap with PD, PSP is pathologically characterized by 4-repeat tau deposits, mainly in the basal ganglia, brainstem, and cerebellum [3], whereas CBS is a clinical syndrome with a heterogeneous underlying neuropathology, represented mainly by tauopathies but also synucleinopathies $[4,5]$. Neuropathological studies showed that most of the cases misdiagnosed as PD are PSP [6-11]. Early differentiation and accurate in vivo diagnosis are important for adequate clinical management and patients' care as well as recruitment in clinical trials of potential disease-modifying therapies. Therefore, there is an urgent need of reliable markers helping the differential diagnosis among the various parkinsonism subtypes.

Our aim here was to investigate the presence of cutaneous p-syn deposits in patients with clinical diagnosis of parkinsonism usually due to tauopathy, i.e., progressive supranuclear palsy (PSP) and corticobasal syndrome (CBS), and to explore the potential utility of this approach in supporting the clinical diagnosis.

\section{MATERIALS AND METHODS}

\section{Study population}

We recruited patients fulfilling the clinical diagnostic criteria for PSP [12], CBS [13], and PD [2] from May 2014 to April 2017 at the Bellaria Hospital of Bologna. All subjects underwent a general and neurological history collection and a detailed neurologic examination. Parkinsonian symptoms severity was rated by UPDRS part III and the stage by the Hoehn and Yahr (H\&Y) scale during the off stage.

Twenty-six healthy individuals were included as controls. Three PD patients [14] and 25 controls had been previously included in other studies $[15,16]$. PD patients were selected from our local database based on high diagnostic accuracy (e.g., fulfilling clinical diagnosis and specific diagnostic tests) to match the number of PSP/CBS patients group. Controls were collected in the context of a broader study on skin biopsy in patients with different neurodegenerative disorders.
The procedures used were approved by the local Human Ethics Committee. According to the Declaration of Helsinki, all subjects gave their written informed consent to the study.

\section{Skin phosphorylated-synuclein deposits}

Punch biopsies $(3 \mathrm{~mm}$ ) were taken from proximal and distal hairy skin sites. Two samples were taken in each skin site 3-4 cm away, to increase the diagnostic accuracy of p-syn deposits [17]. The proximal site included the cervical C7 paravertebral area (close to the spinal ganglia), whereas distal sites were the thigh $(15 \mathrm{~cm}$ above the patella) and distal leg $(10 \mathrm{~cm}$ above the lateral malleolus). As previously described [18], skin samples were immediately fixed overnight in cold Zamboni's fixative and sections cut at $10-\mu \mathrm{m}$ thickness using a cryostat (HM550, Thermo Scientific, Waltham, MA).

Skin sections were double-immunostained overnight with a panel of primary antibodies including rabbit monoclonal p-syn at Ser 129 (p-syn; $1: 500$, Abcam, cat. num. ab-51253) or mouse psyn $(1: 4000$, BioLegend, San Diego, CA, cat. no. 825701), mouse or rabbit PGP. Sections were then washed and further incubated with secondary antibodies (i.e., anti-mouse Alexa Fluor(R) 488 or rabbit cyanine dye fluorophores 3.18 ) for $1 \mathrm{~h}$ at room temperature. Sections were washed and coverslips mounted using fluorescent mounting media. Three skin sections were analyzed for each sample (6 sections for each skin site). Sections were initially viewed and analyzed under a Zeiss fluorescent microscope. The correspondence between p-syn and PGP staining helped to verify the intraneuronal deposits excluding possible non-specific staining and background signal. This analysis showing a high intra-laboratory reproducibility [19] was made blindly to the subjects' status. An operator (AI), blinded to the diagnosis, assigned to each patient samples a code that was used through the skin biopsy analysis phase. P-syn staining was evaluated in each skin sample at high magnification (40X). For 3D colocalization analysis of p-syn with PGP, digital images were acquired using a laser-scanning confocal microscope (Leica DMIRE 2, TCS SL, Leica Microsystems, Heidelberg, Germany). Each image was collected in successive frames of $1-2-\mu \mathrm{m}$ increments on a Z-stack plan at the appropriate wavelengths for the fluorophores coupled with secondary antibodies with a $20 \mathrm{X}$ or $40 \mathrm{X}$ plan apochromat objective and subsequently projected to obtain a double 
Table 1

Demographic and clinical data of patients and controls

\begin{tabular}{|c|c|c|c|c|c|c|}
\hline Subjects & Total & $\mathrm{M} / \mathrm{F}$ & $\begin{array}{c}\text { Age at } \\
\text { biopsy }(y) \\
(\text { mean } \pm \text { SD) }\end{array}$ & $\begin{array}{c}\text { Disease } \\
\text { duration }(\mathrm{y}) \\
(\text { mean } \pm \mathrm{SD})\end{array}$ & $\begin{array}{c}\text { UPDRS part III } \\
(\text { mean } \pm \text { SD) }\end{array}$ & $\begin{array}{c}\text { H\&Y stage, } \\
\text { median } \\
\text { (range) }\end{array}$ \\
\hline $\mathrm{PSP} / \mathrm{CBS}$ & $26(\mathrm{PSP}=18 ; \mathrm{CBS}=8)$ & $8 / 18$ & $70.9 \pm 5.5$ & $3 \pm 2.3$ & $35.5 \pm 10.4$ & $3(2-5)$ \\
\hline PD & 26 & $8 / 18$ & $71.7 \pm 7.6$ & $5.2 \pm 4.8$ & $27.7 \pm 10.8$ & $2.25(1.5-4)$ \\
\hline Controls & 26 & $\begin{array}{c}17 / 9 \\
p=0.008^{*} \\
\left(\chi^{2}\right)\end{array}$ & $\begin{array}{c}67 \pm 10.9 \\
p=0.07 \\
\text { (ANOVA) }\end{array}$ & $\begin{array}{c}/ \\
p=0.106 \\
\text { (M-W test) }\end{array}$ & $\begin{array}{c}\text { / } \\
p=0.02^{*} \\
(\mathrm{M}-\mathrm{W} \text { test })\end{array}$ & $\begin{array}{c}/ \\
p=0.005^{*} \\
\text { (M-W test) }\end{array}$ \\
\hline
\end{tabular}

CBS, corticobasal syndrome; F, female; M, male; M-W, Mann-Whitney test; PD, Parkinson's disease; PSP, progressive supranuclear Palsy; y, years. *significant value.

stained 3D digital image using a computerized system (LCS lite, Leica Microsystems).

\section{Statistical analysis}

Statistical analyses were performed using SPSS 24.0 for Windows. For the analysis of categorical variables (sex), we used Chi-square test. For the analysis of continuous variables, we used Kolmogorov-Smirnov test to verify the normal distribution of the data and to choose between parametric or non-parametric tests. One-way analysis of variance (ANOVA) was used for age. Mann-Whitney U-test was used to compare disease duration, UPDRS and $H \& Y$ values. For all analyses, significance was assumed for corrected $p<0.05$.

\section{RESULTS}

\section{Clinical data}

We included 52 patients (16 M, 36 F). Among them, 18 fulfilled clinical diagnostic criteria of PSP and 8 of CBS, and 26 patients were diagnosed with PD. Demographics and clinical data are summarized in Table 1. Gender distribution was different between groups, with female predominance in the patient group and male predominance in the control group ( $p=0.008$, Chi-square). No significant difference was found in age distribution among groups ( $p=0.07$, ANOVA). Disease duration was similar between PSP/CBS and PD patients, but PSP/CBS cases showed higher scores at UPDRS part III ( $p=0.02$, Mann-Whitney test $)$ and H\&Y stage $(p=0.005$, Mann-Whitney test) compared to PD.

\section{Skin phosphorylated-synuclein deposits}

Irrespectively of the skin biopsy site, skin p-syn deposits were present in only two of the PSP/CBS patients and none of the HC. Conversely, all PD patients showed p-syn deposition in at least one skin sample in at least one site. Specifically, 9 (34.6\%) patients were positive in all the three explored sites, $8(30.7 \%)$ were positive in at least 2 ( 6 at $\mathrm{C} 7$ and thigh and 2 at $\mathrm{C} 7$ and at the leg) and $9(34.6 \%)$ in at least one (5 only at C7, 3 only at the leg and 1 only at the thigh). Deposits were mostly located in skin autonomic nerves around annexes and/or around blood vessels (Fig. 1) ( $p<0.001$, Chi-square).

We further evaluated the distribution of the deposits in each skin site and the concordance between the findings in the two samples collected at each site to investigate if one site was more commonly positive than others and if the analysis of two samples per site increased the sensitivity of the analysis. In the PD group, positive concordant results (i.e., presence of p-syn deposits) between the two samples collected at each site, ensuring a positive finding also when analyzing a single sample, were observed in only 5 cases $(19.2 \%)$ at the leg, $4(15.4 \%)$ at the thigh, and $12(46.1 \%)$ at C7 (Fig. 2A). Discordant results were observed in 9 cases $(34.6 \%)$ at the leg, 12 (46.1\%) at the thigh and $10(38.4 \%)$ at C7. Therefore, the analysis of two samples increased the sensitivity of the analysis from $19.2 \%$ to $53.8 \%$ for the leg site, from $15.4 \%$ to $61.5 \%$ for the thigh and from $46.1 \%$ to $84.5 \%$ for the cervical area. In the PSP/CBS group, one patient showed the presence of p-syn deposits in both the cervical skin samples and the other one in both thigh samples and only one of the cervical samples. In this case, the analysis of a second skin sample did not change the sensitivity of the analysis at the leg or at the thigh whereas increased it from $3.8 \%$ to $7.7 \%$ at the cervical site (Fig. 2B).

Among the two p-syn positive patients in the tauopathies group, one was diagnosed with PSP and the other with CBS. Although clinical and MRI findings supported these diagnoses, both patients had some atypical features for PSP/CBS and more typical of synucleinopathies. 

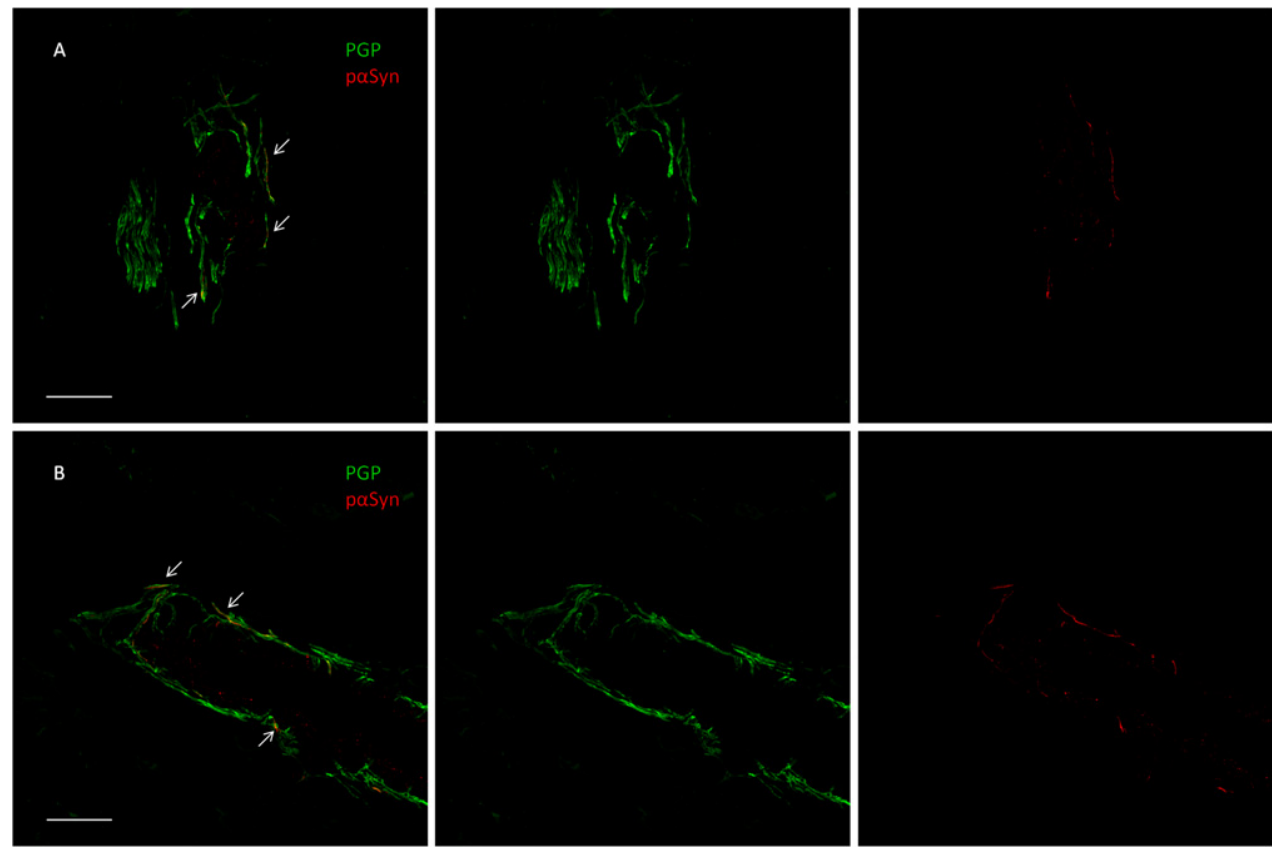

Fig. 1. Confocal microscope study of p-syn deposits in a dermal arteriole of a patient with Parkinson disease (A) and in the patient with CBS (B). In both cases, most of the PGP9.5-stained fibers demonstrate the presence of p-syn as neuritic inclusions (arrowheads) shown by the merged image (scalebar $50 \mu \mathrm{M})$.
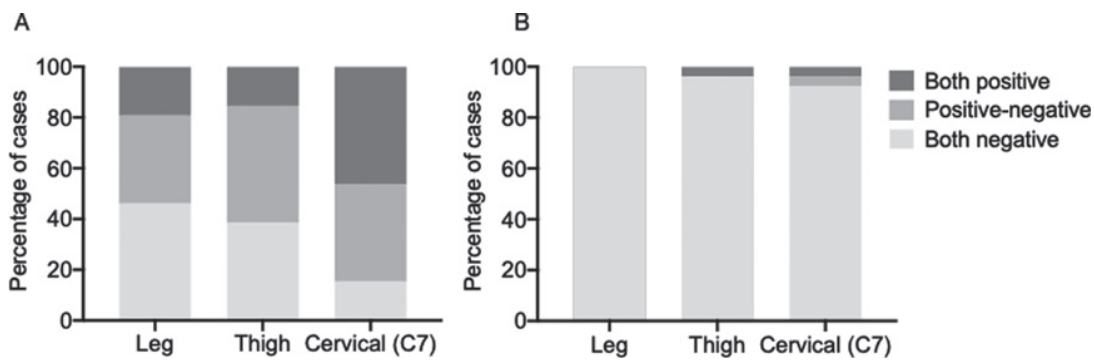

Fig. 2. Concordance of p-syn deposition in two adjacent skin samples at each site in PD (A) and in PSP/CBS (B) patients. The analysis of two samples increased the sensitivity of the analysis.

The PSP patient was an 84-year-old man with a 2-year history of gait impairment, upper limb motor clumsiness and postural instability, leading to several falls. Neurological examination showed supranuclear ophthalmoplegia, hypomimia, bradykinesia prevalent on the left side, limbs rigidity, jerky bilateral hands tremor and postural instability. Brain MRI showed mesencephalon atrophy.

Amantadine therapy improved his symptoms; therefore, he was started on low-dose L-dopa, upon which he developed visual hallucinations and orthostatic hypotension requiring the withdrawal of this therapy.

The CBS patient was a 70-year-old woman, with a 3-year history of limb bradykinesia and dystonia prevalent in the right side associated with limb apraxia. Brain MRI detected bilateral parietal cortical atrophy, mainly in the left side, and ${ }^{18}$ F-fluorodeoxyglucose PET showed left parietal hypometabolism. She had a slow parkinsonism progression, a moderate response to levodopa treatment, and orthostatic hypotension; cardiac denervation was documented by 123I-meta-iodobenzylguanidine myocardial scintigraphy [20].

\section{DISCUSSION}

In this study, we investigated the presence of p-syn deposits in the skin samples of patients with clinically 
similar but neuropathologically different conditions, PD and PSP/CBD. In our cohort, all PD patients had skin p-syn deposits versus only 7.7\% (2/26) of patients with a clinical diagnosis of PSP/CBS and none of the controls, confirming that the detection of skin p-syn deposits may help in the differential diagnosis of parkinsonism. Also, we showed that the analysis of two adjacent skin samples increases the sensitivity of detecting p-syn in skin nerves.

Recently, we [15, 21-24] and others [25] demonstrated the utility of skin nerve phosphorylated- $\alpha$ synuclein (p-syn) deposits as a diagnostic marker for $\mathrm{PD}$ in vivo. A recent metanalysis confirmed the reliability of this biomarker, however, the majority of the studies compared PD with healthy controls (HC) or with patients with other synucleinopathies [26]. Only few studies, on small samples, included patients with atypical parkinsonism due to tauopathy $[21,24$, $27,28]$.

In two previous studies, we included a small proportion of patients with tauopathies, none of them showing p-syn deposits [21, 24]. Another study found the same results in a group of 15 patients [24]. Rodríguez-Leyva et al. [27] investigated the presence of $\alpha$-syn in 10 PSP, 17 PD, and $17 \mathrm{HC}$ and found higher $\alpha$-syn positivity in PD. However, this study used antibodies against total $\alpha$-syn which can show deposits also in normal controls and are considered less specific of misfolded $\alpha$-syn compared to anti-p-syn antibodies.

Our analysis remarked the importance of the site chosen for the biopsy as well as of the number of samples analyzed at each site. We confirmed a higher p-syn positivity rate in the cervical site in PD, as already reported [21, 29]. However, a not negligible proportion of cases, including one PSP/CBS patient, showed the presence of isolated deposits in other sites, in accordance with the known patchy-like deposition of p-syn [15], outlying the utility of investigating at least two sites. The patchy-like distribution justifies, as well, the utility of double samples analysis at each site, which increased the sensitivity of the p-syn analysis.

Interestingly, in our sample, two patients, one diagnosed with CBS and the other with PSP, presented skin p-syn deposits. Both patients presented clinical and radiological criteria supporting these clinical diagnoses, suggesting a false positive result. However, both cases had some atypical features, i.e., a mild response to L-dopa treatment, psychiatric symptoms exacerbation upon carbidopa/levodopa treatment, i.e., visual hallucinations [30], and the presence of cardiac denervation at MIBG scintigraphy, more suggestive of a synucleinopathy [31]. Indeed, several studies showed a significant overlap in clinical and radiological findings between PD and PSP [32]. Similarly, a recent study showed that a small percentage of patients with a neuropathological diagnosis of dementia with Lewy bodies received a clinical diagnosis of CBS [5]. Therefore, also in our cases with supposed tauopathies a misdiagnosis with the presence instead of an underlying synucleinopathy cannot be excluded. Another possibility is the occurrence of a coexisting tau pathology in patients with synucleinopathies or viceversa. A previous study showed that $9.6 \%$ of confirmed PD had concomitant PSP pathological features [33]. On the other hand, the presence of Lewy bodies has been recently reported in $8 \%$ of cases diagnosed with atypical parkinsonism [34], which is in line with our observation.

According to the prion-like spread of $\alpha$-syn, Lewy body pathology can occur in a caudo-rostral manner in primary synucleinopathies. However, Lewy body pathology can also remain limited to certain brain areas, as it is often observed in the amygdala and limbic regions of patients with $\mathrm{AD}$ [35]. This could be due to biochemical differences between $\alpha$-syn aggregates [36]. These differences could be also relevant in the periphery as they could reflect differently on skin biopsy findings and should be further investigated.

Our study has limitations. First, the diagnosis was based on clinical criteria and no pathological confirmation was available. Additional investigations (i.e., neuroimaging findings) and clinical follow-up were used to support the diagnoses, despite the aforementioned weaknesses. Without neuropathology, the meaning of the p-syn deposits finding in two PSP/ CBS cases remains unclear. Our data supported the presence of a synucleinopathy in cases classified as tauopathy according to clinical criteria or alternatively underlined that some patients with tauopathy presented instead a co-pathology [37]. Hence the importance of identifying reliable biomarkers and accumulate in vivo data, relying on the current diagnostic approach which is based on the clinical phenotype. Secondly, we included only few CBS cases. A further limitation could be the lack of inclusion of patients with multiple system atrophy-parkinsonism (MSA-P). However, we have previously reported that intraneural $\mathrm{p}$-syn deposits were found in $72 \%$ of patients with MSA-P, with a different distribution than PD, mainly found in somatic fibers of subepidermal plexi rather than autonomic fibers $[23,38]$. 
Despite these limitations, this is to our knowledge the largest study comparing in vivo the peripheral deposition of misfolded $\alpha$-synuclein in PD, HC, and PSP/CBS cases. We showed that the presence of skin p-syn deposits accurately distinguish patients with PD from those with atypical parkinsonism. Further studies including larger samples of patients are needed to confirm these results.

\section{CONFLICT OF INTEREST}

The authors declare that they have no competing interests.

\section{REFERENCES}

[1] Spillantini MG, Crowther RA, Jakes R, Hasegawa M, Goedert M (1998) $\alpha$-Synuclein in filamentous inclusions of Lewy bodies from Parkinson's disease and dementia with Lewy bodies. Proc Natl Acad Sci U S A 95, 64696473.

[2] Postuma RB, Berg D, Stern M, Poewe W, Olanow CW, Oertel W, Obeso J, Marek K, Litvan I, Lang AE, Halliday G, Goetz CG, Gasser T, Dubois B, Chan P, Bloem BR, Adler CH, Deuschl G (2015) MDS clinical diagnostic criteria for Parkinson's disease. Mov Disord 30, 1591-1601.

[3] Scaravilli T, Tolosa E, Ferrer I (2005) Progressive supranuclear palsy and corticobasal degeneration: Lumping versus splitting. Mov Disord 20 Suppl 12, S21-S28.

[4] Saranza GM, Whitwell JL, Kovacs GG, Lang AE (2019) Corticobasal degeneration, Elsevier Ltd.

[5] Kasanuki K, Josephs KA, Ferman TJ, Murray ME, Koga S, Konno T, Sakae N, Parks A, Uitti RJ, Van Gerpen JA, GraffRadford NR, Wszolek ZK, Dickson DW (2018) Diffuse Lewy body disease manifesting as corticobasal syndrome A rare form of Lewy body disease. Neurology 91, E268-E279.

[6] Adler CH, Beach TG, Hentz JG, Shill HA, Caviness JN, Driver-Dunckley E, Sabbagh MN, Sue LI, Jacobson SA, Belden CM, Dugger BN (2014) Low clinical diagnostic accuracy of early vs advanced Parkinson disease: Clinicopathologic study. Neurology 83, 406-412.

[7] Jankovic J, Rajput AH, McDermott MP, Perl DP (2000) The evolution of diagnosis in early Parkinson disease. Arch Neurol 57, 369-372.

[8] Hughes AJ, Daniel SE, Kilford L, Lees AJ (1992) Accuracy of clinical diagnosis of idiopathic Parkinson's disease: A clinico-pathological study of 100 cases. J Neurol Neurosurg Psychiatry 55, 181-184.

[9] Hughes AJ, Daniel SE, Lees AJ (2001) Improved accuracy of clinical diagnosis of Lewy body Parkinson's disease. Neurology 57, 1497-1499.

[10] Rizzo G, Copetti M, Arcuti S, Martino D, Fontana A, Logroscino G (2016) Accuracy of clinical diagnosis of Parkinson disease. Neurology 86, 566-576.

[11] Beach TG, Adler CH (2018) Importance of low diagnostic Accuracy for early Parkinson's disease. Mov Disord 33, $1551-1554$.

[12] Litvan I, Agid Y, Jankovic J, Goetz C, Brandel JP, Lai EG, Wenning G, D'Olhaberriague L, Verny M, Chaudhuri KR, McKee A, Jellinger K, Bartko JJ, Mangone CA, Pearce RKB
(1996) Accuracy of clinical criteria for the diagnosis of progressive supranuclear palsy (Steele-Richardson-Olszewski syndrome). Neurology 46, 922-930.

[13] Armstrong MJ, Litvan I, Lang AE, Bak TH, Bhatia KP, Borroni B, Boxer AL, Dickson DW, Grossman M, Hallett M, Josephs KA, Kertesz A, Lee SE, Miller BL, Reich SG, Riley DE, Tolosa E, Tröster AI, Vidailhet M, Weiner WJ (2013) Criteria for the diagnosis of corticobasal degeneration. $\mathrm{Neu}$ rology 80, 496-503.

[14] Donadio V, Incensi A, Rizzo G, Scaglione C, Capellari S, Fileccia E, Avoni P, Liguori R. (2017) Spine topographical distribution of skin $\alpha$-synuclein deposits in idiopathic Parkinson disease. J Neuropathol Exp Neurol 76, 384-389

[15] Donadio V, Incensi A, Piccinini C, Cortelli P, Giannoccaro MP, Baruzzi A, Liguori R (2016) Skin nerve misfolded $\alpha$ synuclein in pure autonomic failure and Parkinson disease. Ann Neurol 79, 306-316.

[16] Donadio V, Incensi A, Rizzo G, Capellari S, Pantieri R, Stanzani Maserati M, Devigili G, Eleopra R, Defazio G, Montini F, Baruzzi A, Liguori R (2017) A new potential biomarker for dementia with Lewy bodies: Skin nerve $\alpha$ synuclein deposits. Neurology 89, 318-326.

[17] Donadio V (2019) Skin nerve $\alpha$-synuclein deposits in Parkinson's disease and other synucleinopathies: a review. Clin Auton Res 29, 577-585.

[18] Donadio V, Incensi A, Giannoccaro MP, Cortelli P, Di Stasi V, Pizza F, Jaber MA, Baruzzi A, Liguori R (2012) Peripheral autonomic neuropathy: Diagnostic contribution of skin biopsy. J Neuropathol Exp Neurol 71, 1000-1008.

[19] Donadio V, Doppler K, Incensi A, Kuzkina A, Janzen A, Mayer G, Volkmann J, Rizzo G, Antelmi E, Plazzi G, Sommer C, Liguori R, Oertel WH (2019) Abnormal $\alpha$-synuclein deposits in skin nerves: intra- and inter-laboratory reproducibility. Eur J Neurol 26, 1245-1251.

[20] Infante R, Incensi A, Rizzo G, Donadio V, Liguori R (2019) Editors' note: Diffuse Lewy body disease manifesting as corticobasal syndrome: A rare form of Lewy body disease. Neurology 93, 411.

[21] Donadio V, Incensi A, Leta V, Giannoccaro MP, Scaglione C, Martinelli P, Capellari S, Avoni P, Baruzzi A, Liguori R (2014) Skin nerve a-synuclein deposits A biomarker for idiopathic Parkinson disease. Neurology 82, 1362-1369.

[22] Donadio V, Incensi A, El-Agnaf O, Rizzo G, Vaikath N, Del Sorbo F, Scaglione C, Capellari S, Elia A, Stanzani Maserati M, Pantieri R, Liguori R (2018) Skin $\alpha$-synuclein deposits differ in clinical variants of synucleinopathy: an in vivo study. Sci Rep 8, 14246.

[23] Giannoccaro MP, Donadio V, Giannini G, Devigili G, Rizzo G, Incensi A, Cason E, Calandra-Buonaura G, Eleopra R, Cortelli P, Liguori R (2020) Comparison of 123I-MIBG scintigraphy and phosphorylated $\alpha$-synuclein skin deposits in synucleinopathies. Parkinsonism Relat Disord 81, 48-53.

[24] Donadio V, Wang Z, Incensi A, Rizzo G, Fileccia E, Vacchiano V, Capellari S, Magnani M, Scaglione C, Stanzani Maserati M, Avoni P, Liguori R, Zou W (2021) In vivo diagnosis of synucleinopathies: a comparative study of skin biopsy and RT-QuIC. Neurology 96, e2513-e2524.

[25] Doppler K, Ebert S, Üçeyler N, Trenkwalder C, Ebentheuer J, Volkmann J, Sommer C (2014) Cutaneous neuropathy in Parkinson's disease: A window into brain pathology. Acta Neuropathol 128, 99-109.

[26] Tsukita K, Sakamaki-Tsukita H, Tanaka K, Suenaga T, Takahashi R (2019) Value of in vivo $\alpha$-synuclein deposits in Parkinson's disease: A systematic review and meta-analysis. Mov Disord 34, 1452-1463. 
[27] Rodríguez-Leyva I, Chi-Ahumada EG, Carrizales J, Rodríguez-Violante M, Velázquez-Osuna S, Medina-Mier V, Martel-Gallegos MG, Zarazúa S, Enríquez-Macías L, Castro A, Calderón-Garcidueñas AL, Jiménez-Capdeville ME (2016) Parkinson disease and progressive supranuclear palsy: Protein expression in skin. Ann Clin Transl Neurol 3, 191-199.

[28] Doppler K, Weis J, Karl K, Ebert S, Ebentheuer J, Trenkwalder C, Klebe S, Volkmann J, Sommer C (2015) Distinctive distribution of phospho-alpha-synuclein in dermal nerves in multiple system atrophy. Mov Disord 30, 1688-1692.

[29] Melli G, Vacchi E, Biemmi V, Galati S, Staedler C, Ambrosini R, Kaelin-Lang A (2018) Cervical skin denervation associates with alpha-synuclein aggregates in Parkinson disease. Ann Clin Transl Neurol 5, 1394-1407.

[30] Gomperts SN (2016) Lewy body dementia. Contin (Minneap Minn) 22, 435-463.

[31] Giannoccaro MP, Donadio V, Incensi A, Pizza F, Cason E, Di Stasi V, Martinelli P, Scaglione C, Capellari S, Treglia G, Liguori R (2015) Skin biopsy and I-123 MIBG scintigraphy findings in idiopathic Parkinson's disease and parkinsonism: A comparative study. Mov Disord 30, 986-989.

[32] Alster P, Madetko N, Koziorowski D, Friedman A (2020) Progressive supranuclear palsy-parkinsonism predominant (PSP-P) - a clinical challenge at the boundaries of PSP and Parkinson's disease (PD). Front Neurol 11, 180.

[33] Dugger BN, Adler CH, Shill HA, Caviness J, Jacobson S, Driver-Dunckley E, Beach TG (2014) Concomitant pathologies among a spectrum of parkinsonian disorders. Parkinsonism Relat Disord 20, 525-529.

[34] Geut H, Hepp DH, Foncke E, Berendse HW, Rozemuller JM, Huitinga I, van de Berg WDJ (2020) Neuropathological correlates of parkinsonian disorders in a large Dutch autopsy series. Acta Neuropathol Commun 8, 39.

[35] Toledo JB, Gopal P, Raible K, Irwin DJ, Brettschneider J, Sedor S, Waits K, Boluda S, Grossman M, Van Deerlin VM, Lee EB, Arnold SE, Duda JE, Hurtig H, Lee VM, Adler CH, Beach TG, Trojanowski JQ (2016) Pathological $\alpha$-synuclein distribution in subjects with coincident Alzheimer's and Lewy body pathology. Acta Neuropathol 131, 393-409.

[36] Sorrentino ZA, Goodwin MS, Riffe CJ, Dhillon JS, Xia Y, Gorion KM, Vijayaraghavan N, McFarland KN, Golbe LI, Yachnis AT, Giasson BI (2019) Unique $\alpha$-synuclein pathology within the amygdala in Lewy body dementia: implications for disease initiation and progression. Acta Neuropathol Commun 7, 142.

[37] Muccioli L, Mammana A, Incensi A, Baiardi S, Parchi P, Liguori R, Donadio V (2021) The in vivo diagnosis of concomitant Alzheimer and Lewy body pathology: a case report. J Neuropathol Exp Neurol, doi: 10.1093/jnen/ nlab065.

[38] Donadio V, Incensi A, Rizzo G, De Micco R, Tessitore A, Devigili G, Del Sorbo F, Bonvegna S, Infante R, Magnani M, Zenesini C, Vignatelli L, Cilia R, Eleopra R, Tedeschi G, Liguori R (2020) Skin biopsy may help to distinguish multiple system atrophy-parkinsonism from Parkinson's disease with orthostatic hypotension. Mov Disord 35, 1649-1657. 